\title{
Hemorheologic assessment for screening diabetic nephropathy in type 2 diabetes
}

*Sangbae Lee, Jung Hye Kim, Kahui Park, Min Jin Kim, Ji sun Nam, Jong Suk Park, Chul Woo Ahn, Kyung Rae Kim

Gangnam Severance Hospital, Division of endocrinology Seoul, Korea

\section{Background and Aim}

Hemorheologic alterations or changes in blood viscosity have been suggested to play a role in the pathogenesis of diabetic microvascular complications.

We measured various hemorheologic parameters in type 2 diabetes patients and assessed their possible role as a diagnostic tool for diabetic

\section{Methods}

Four hundred-seventy patients with type 2 diabetes were included in this study. Hemorheologic parameters, including erythrocyte deformability, elongation index (EI), critical shear stress (CSS), and aggregation index (AI) were measured using microfluidic hemorheometer. Various metabolic parameters were assessed from fasting blood samples and urinary albumin to creatinine ratio was used to assess diabetic nephropathy.

\section{Results}

Table 1. Clinical characteristics and hemorheologic parameters of subjects according to urinary ACR group

\begin{tabular}{|c|c|c|c|c|}
\hline & \multicolumn{3}{|c|}{ ACR group } & \multirow{3}{*}{ P-value } \\
\hline & $<30$ & $30 \sim 300$ & $>300$ & \\
\hline & $n=375$ & $n=55$ & $n=40$ & \\
\hline AGE (years) & $55.48(10.31)$ & $59.18(10.81)$ & $61.53(9.30)$ & 0.000 \\
\hline BMI $\left(\mathbf{k g} / \mathbf{m}^{2}\right)$ & 24.20 (2.98) & 24.18 (3.41) & $26.01(4.10)$ & 0.002 \\
\hline SBP $(\mathrm{mmHg})$ & $121.63(8.87)$ & 121.83 (11.99) & 132.09 (12.98) & 0.000 \\
\hline $\mathrm{DBP}(\mathrm{mmHg})$ & $73.58(5.90)$ & $73.01(8.94)$ & 77.85 (7.47) & 0.001 \\
\hline HbA1c (\%) & $6.31(1.34)$ & $8.29(1.81)$ & $8.22(1.62)$ & 0.000 \\
\hline BUN (mg/dL) & $14.74(7.29)$ & $19.17(10.41)$ & $28.12(11.08)$ & 0.000 \\
\hline $\mathrm{Cr}(\mathrm{mg} / \mathrm{dL})$ & $0.77(0.25)$ & $0.98(055)$ & $1.57(0.71)$ & 0.000 \\
\hline eGFR $\left(\mathrm{ml} / \mathrm{min} / \mathrm{m}^{2}\right)$ & 84.74 (10.33) & $73.96(21.00)$ & 49.95 (22.49) & 0.000 \\
\hline $\mathrm{TC}(\mathrm{mg} / \mathrm{dL})$ & $190.89(41.32)$ & $176.26(38.61)$ & $179.60(49.82)$ & 0.022 \\
\hline $\mathrm{TG}(\mathrm{mg} / \mathrm{dL})$ & $131.14(76.45)$ & $177.10(112.29)$ & $140.72(72.48)$ & 0.000 \\
\hline LDL-C (mg/dL) & $116.21(33.67)$ & $97.38(37.11)$ & 101.40 (37.95) & 0.000 \\
\hline HDL-C (mg/dL) & $50.30(13.10)$ & $42.87(10.08)$ & $43.80(11.73)$ & 0.000 \\
\hline $\operatorname{ESR}(\mathrm{mm} / \mathrm{h})$ & 14.72 (12.57) & 27.40 (22.35) & 40.52 (23.59) & 0.000 \\
\hline Fibrinogen (mg/dL) & $271.23(56.19)$ & 313.28 (78.68) & 334.20 (81.34) & 0.000 \\
\hline El at $1.5 \mathrm{~Pa}(\%)$ & $0.2180(0.0608)$ & $0.2148(0.0425)$ & $0.2160(0.0182)$ & 0.913 \\
\hline $\mathrm{El}$ at $3 \mathrm{~Pa}(\%)$ & $0.3215(0.0180)$ & $0.3181(0.0186)$ & $0.3161(0.0150)$ & 0.102 \\
\hline El max (\%) & $0.5575(0.1863)$ & $0.5616(0.0129)$ & $0.5599(0.0138)$ & 0.238 \\
\hline Critical time (sec) & $8.90(3.55)$ & $7.93(3.11)$ & $6.53(1.36)$ & 0.000 \\
\hline Critical shear stress ( $\mathrm{Pa}$ & 261.82 (129.97) & $308.90(170.12)$ & $345.88(154.41)$ & 0.000 \\
\hline $\mathrm{Al}(\%)$ & $40.41(9.36)$ & 42.05 (9.50) & 41.09 (7.92) & 0.454 \\
\hline $\begin{array}{l}\text { Fibrinogen/3EI } \\
(\mathrm{mg} / \mathrm{dL} \%)\end{array}$ & $811.73(245.70)$ & $967.99(273.47)$ & $1060.69(268.75)$ & 0.000 \\
\hline $\begin{array}{l}\text { CSS/3EI } \\
(\mathrm{Pa} / \%)\end{array}$ & $788.18(454.75)$ & $985.92(615.56)$ & $1106.44(531.97)$ & 0.000 \\
\hline $\begin{array}{l}\text { Fibrinogen/CSS } \\
\text { (mg/dLPa) }\end{array}$ & 788.18 (454.75) & $985.92(615.56)$ & 1106.44 (531.97) & 0.000 \\
\hline
\end{tabular}

Data are means $\pm S D$

Abb: BMI, body mass index; SBP, systolic blood pressure; DBP, diastolic blood pressure; HbA1c, hemoglobin A1c; BUN, Blood urea nitrogen; $\mathrm{Cr}$, creatinine; eGFR, estimated glomerular filtration rate; TC, total cholesterol; TG, triglyceride; LDL-C, lowdensity lipoprotein cholesterol; HDL-C, high-density lipoprotein cholesterol; ESR erythrocyte sedimentation rate; El. elongation index; Al, aggregation index; $3 \mathrm{EI}, \mathrm{El}$ a $3 \mathrm{~Pa}$; CSS, critical shear stress

\section{Results}

Table 2. Hemorheologic parameters of subjects according to eGFR group

\begin{tabular}{|c|c|c|c|c|c|}
\hline & \multicolumn{4}{|c|}{ CKD stage } & \multirow{3}{*}{ P-value } \\
\hline & $\begin{array}{c}\text { Group IV } \\
(\text { eGFR<30) }\end{array}$ & $\begin{array}{c}\text { Group III } \\
(30 \leq e G F R<60)\end{array}$ & $\begin{array}{c}\text { Group II } \\
(60 \leq \text { eGFR }<90)\end{array}$ & $\begin{array}{c}\text { Group I } \\
(e G F R>90)\end{array}$ & \\
\hline & $n=10$ & $\mathrm{n}=48$ & $n=142$ & $\mathrm{n}=260$ & \\
\hline $\begin{array}{c}\text { Fibrinogen } \\
\text { (mg/dL) }\end{array}$ & $\begin{array}{l}357.80 \\
(77.52)\end{array}$ & $\begin{array}{l}311.98 \\
(66.00)\end{array}$ & $\begin{array}{l}284.26 \\
(65.60)\end{array}$ & $\begin{array}{l}271.43 \\
(60.34)\end{array}$ & 0.000 \\
\hline $\begin{array}{c}\text { El at } 1.5 \mathrm{~Pa} \\
(\%)\end{array}$ & $\begin{array}{c}0.2106 \\
(0.0172)\end{array}$ & $\begin{array}{l}0.2110 \\
(0.0517)\end{array}$ & $\begin{array}{l}0.2198 \\
(0.0303)\end{array}$ & $\begin{array}{c}0.2177 \\
(0.0678)\end{array}$ & 0.790 \\
\hline $\begin{array}{c}\text { El at } 3 \mathrm{~Pa} \\
(\%)\end{array}$ & $\begin{array}{l}0.3106 \\
(0.0169)\end{array}$ & $\begin{array}{l}0.3169 \\
(0.0153)\end{array}$ & $\begin{array}{l}0.3190 \\
(0.0182)\end{array}$ & $\begin{array}{c}0.3226 \\
(0.0181)\end{array}$ & 0.021 \\
\hline $\begin{array}{l}\text { Critical shear } \\
\text { Stress } \\
(\mathrm{Pa})\end{array}$ & $\begin{array}{l}323.08 \\
(82.48)\end{array}$ & $\begin{array}{l}287.53 \\
(144.46)\end{array}$ & $\begin{array}{l}274.75 \\
(162.83)\end{array}$ & $\begin{array}{l}270.10 \\
(125.92)\end{array}$ & 0.571 \\
\hline $\begin{array}{l}\text { CSS/3EI } \\
(\mathrm{Pa} / \%)\end{array}$ & $\begin{array}{l}1043.23 \\
(272.76)\end{array}$ & $\begin{array}{c}908.50 \\
(459.26)\end{array}$ & $\begin{array}{l}849.13 \\
(558.13)\end{array}$ & $\begin{array}{c}811.53 \\
(463.59)\end{array}$ & 0.289 \\
\hline $\begin{array}{l}\text { Fibrinogen/3EI } \\
\text { (mg/dL\%) }\end{array}$ & $\begin{array}{l}1040.89 \\
(430.22)\end{array}$ & $\begin{array}{l}987.86 \\
(221.59)\end{array}$ & $\begin{array}{l}862.61 \\
(262.95)\end{array}$ & $\begin{array}{l}808.86 \\
(254.72)\end{array}$ & 0.000 \\
\hline $\begin{array}{l}\text { Critical time } \\
\quad(\mathrm{sec})\end{array}$ & $\begin{array}{c}5.66 \\
(0.88)\end{array}$ & $\begin{array}{c}8.71 \\
(6.27)\end{array}$ & $\begin{array}{c}8.90 \\
(3.09)\end{array}$ & $\begin{array}{c}8.51 \\
(2.87)\end{array}$ & 0.024 \\
\hline
\end{tabular}

Data are means \pm SD

Abb: El. elongation index; $\mathrm{Al}$, aggregation index; $3 \mathrm{EI}$, El at $3 \mathrm{~Pa}$; CSS, critical shear stress

Table 3. Multiple regression analysis for urinary ACR

\begin{tabular}{|c|c|c|c|c|c|}
\hline \multirow{2}{*}{$\begin{array}{l}\text { Independent } \\
\text { variable }\end{array}$} & \multicolumn{5}{|c|}{ Urine ACR } \\
\hline & B & SE & B & $\mathrm{T}$ & P-value \\
\hline Constant & -225.55 & 513.09 & & -0.44 & 0.660 \\
\hline Age & -5.143 & 3.690 & -0.61 & -1.394 & 0.164 \\
\hline ESR & 9.327 & 2.946 & 0.182 & 3.166 & 0.002 \\
\hline Het & -33.493 & 9.900 & -0.175 & -3.383 & 0.001 \\
\hline BMI & 45.864 & 12.103 & 0.166 & 3.789 & 0.000 \\
\hline HbA1c & 76.623 & 24.261 & 0.142 & 3.158 & 0.002 \\
\hline Fibrinogen/3EI & 0.347 & 0.171 & 0.101 & 2.026 & 0.043 \\
\hline
\end{tabular}

Abb: $B$, unstandardized regression coefficient: $B$, standardized $B$ : ESR, erythrocyte sedimentation rate; Hct, hematocrit: BMI, Body mass index; HbA1c, hemoglobin A1c; $3 \mathrm{EI}, \mathrm{El}$ at $3 \mathrm{~Pa}$

Figure 1. ROC curve between Fibrinogen/3EI and microalbuminuria

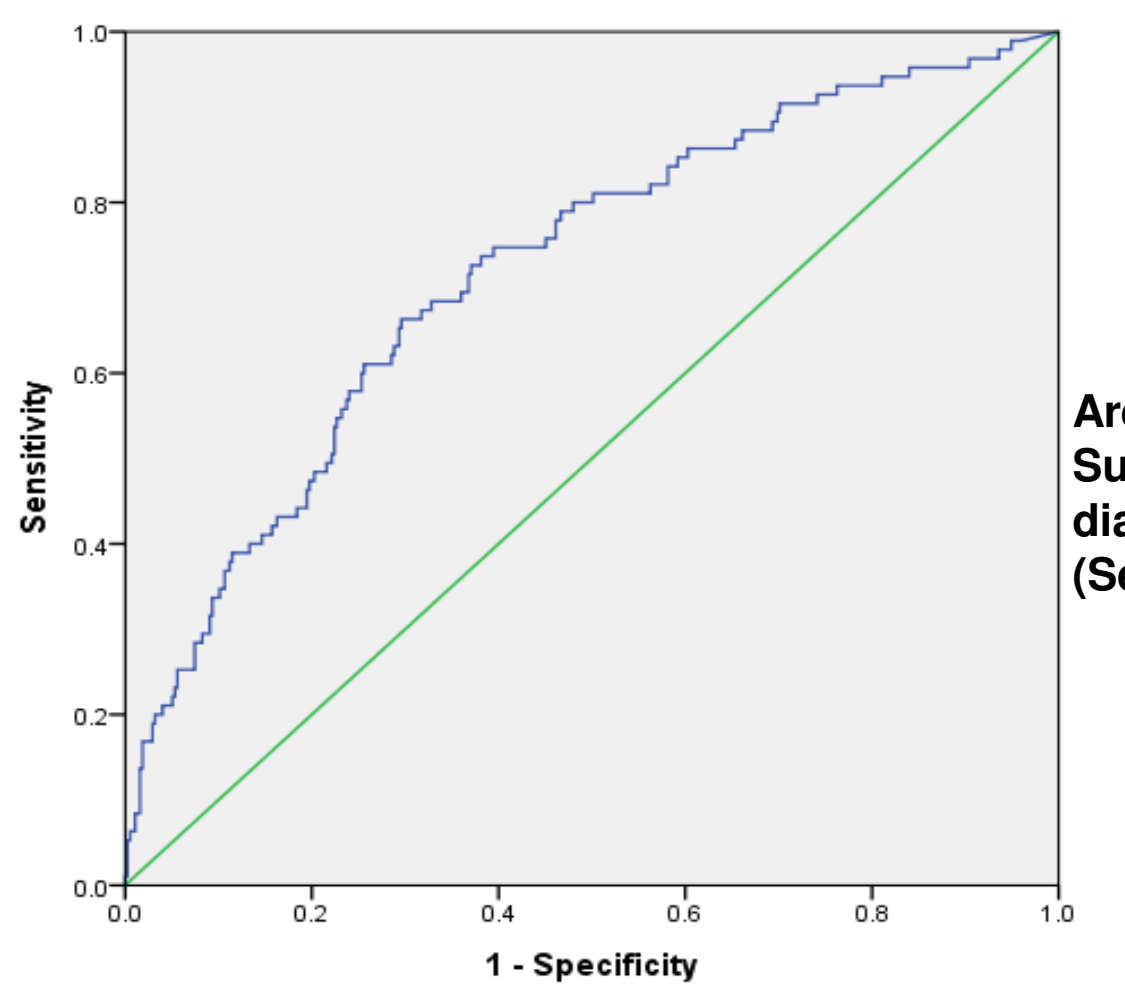

Area under curve (AUC): 0.721 Suggesting cut off value for diabetic nephropathy: $860 \mathrm{mg} / \mathrm{dL} \%$ (Sensitivity-74\%, Specificity - 62\%)

\section{Conclusion}

Fibrinogen/El is a sensitive parameter measured via point-ofcare testing for screening diabetic nephropathy in patients with type 2 diabetes. 